\title{
Transitions in Interface Objects: Searching Databases
}

\author{
Tim Gamble ${ }^{1}$ and Jon May $^{2}$ \\ ${ }^{1}$ Department of Psychology, University of Winchester, Winchester SO22 4NR, UK \\ ${ }^{2}$ School of Psychology, University of Plymouth, Plymouth PL4 8AA, UK \\ Correspondence should be addressed to Tim Gamble; tim.gamble@winchester.ac.uk
}

Received 5 November 2015; Accepted 5 May 2016

Academic Editor: Jean Vanderdonckt

Copyright (C) 2016 T. Gamble and J. May. This is an open access article distributed under the Creative Commons Attribution License, which permits unrestricted use, distribution, and reproduction in any medium, provided the original work is properly cited.

\begin{abstract}
Two experiments demonstrate that a list-like database interface which benefits from the persistence of contextual information does not show the same degree of benefit of collocating objects over display changes that has been previously observed in a map-searching study. This provides some support for the claim that the nature of the task must be taken into account in choosing how to design dynamic displays. We discuss the benefit of basing design principles on theoretical models derived from film cutting methods used in cinematography, so that they can be extended to novel design situations.
\end{abstract}

\section{Introduction}

It has been argued that cinematography can provide inspiration for HCI designers, who could adopt methods used by film makers [1]. Designers would benefit from a theoretically based analysis of why film conventions work (e.g., [2, 3]), so that they could base interface design decisions on this understanding, rather than applying them in a trial and error manner. May et al. [4] tested a cognitive model of film perception [5] inspired by Barnard's Interacting Cognitive Subsystems (ICS) model [6] and showed that within scenes film editors ensure that the object viewers have been looking at does not grossly change screen location over cuts ("collocation"), whereas at a narrative break over space or time at the end of scenes, viewers are often required to search the screen for a new topic ("translation"). May and Gamble [7] demonstrated that, in computerised map interfaces, a "filmic" version that used collocation (where the map zoomed underneath the cursor so that the geographical location that had been clicked remained in the same place) was easier to use than an "unfilmic" version that used translation (where the location clicked was moved to the centre of the screen as it zoomed in). Collocation reduced both the eye movements and interaction duration. There was some indication that collocation did not always improve performance, such as in the final step of the task when the search was simple, pointing to the need to consider the task context in applying design guidelines.

May and Gamble [7] suggested that collocating interface objects may not be appropriate where structural changes in the display could guide the user to novel information, when context is important or when any translation of focal point after a screen change is predictable. In cinematography, for example, May et al. [4] noted that film editors would place close-ups of characters having a conversation on opposite sides of the screen, so that the viewers' gaze had to move predictably from side to side across film cuts as if both actors were physically present. They suggested that tasks that might benefit from the spatial movements required in translations over display changes might include searching through hierarchical databases, where, successively, refinements of the category within which one is searching imply a predictable direction for changes in gaze location. Instead of replacing the initial set of categories with a second, finergrained subset, a translated interface could place the new subset off to one side, with the original selection still visible so that the result of the interaction is contextualised by the superordinate category name. This has long been used in Apple's iTunes ${ }^{\mathrm{TM}}$ software, where three panes arranged horizontally contain progressively more detailed genre, artist, 
and album information, in hierarchical order. By selecting an item in one pane, contextually relevant information is displayed in the next pane to the right.

In contrast to this theoretical account that links visual search and task structure, it could be argued that collocation of screen objects over interface transitions would always be beneficial, simply because it minimises eye movements. In this paper, we show that this is not clearly the case. We compared two current database designs: a translated design, where new information appears alongside previous windows, and a collocated design, where new information replaces the previous window. If collocation improved map interfaces [7] simply by minimising eye movements, then the collocated versions of the databases should benefit on every task step, because the new information is physically located close to the previous focus of attention. On the other hand, if collocation worked because it was concordant with the users' task, then it should not be as useful here, because the database task involves successively moving through a data structure in a predictable manner, with detail unfolding on each operation. Despite minimising the distance between targets within the interface, the collocated design does not match the task structure as well as a translated design, because it removes the sense of moving successively through the data while retaining the availability of contextual information.

\section{Experiment 1}

Method. Twenty-six participants, all students at a large UK university (aged 18 to 27; mean 20.5 years; 23 females), were told that they would be searching for ten items in each of two databases (car parts and music). On each trial, they had to locate and select either a part for a specific car from a specific maker or an album by an artist in a particular genre; for example, "Please order part number: CC05 for model: Seicento for make: Fiat" or "Please order album: 05AT for artist: Nina Simone from Genre: Jazz."

Each trial required participants to locate the appropriate car maker or musical genre from a list of ten options, click to select it, and then click an $\mathrm{OK}$ button in the lower right of the window. A second window containing the car types or artists opened, with a Back button and an $\mathrm{OK}$ button. On selecting the appropriate item and clicking the OK button, the third and final window opened, displaying ten alphanumerical codes, again with Back and OK buttons. After selecting an item and clicking $\mathrm{OK}$, the trial ended with either a confirmation of correct ordering or an error message. The next trial began when this message was dismissed by clicking OK.

The three list windows each measured $120 \mathrm{~mm}$ wide by $100 \mathrm{~mm}$ high. The first window was always located in the left third of the screen (which was $405 \mathrm{~mm}$ wide), centred vertically. The list items were displayed in Helvetica font, with capitals $4 \mathrm{~mm}$ high. In the translated interface, the second window was positioned in the middle third of the screen, and the third window was positioned in the right third of the screen (Figure 1). In the collocated interface, each window opened in the left third of the screen, with the previous window(s) moving to the right, so that on the final step they were in the reverse order to the translated interface.

Participants completed both the collocated and translated tasks, with the order of interface design being balanced over participants and the task content (music or car parts) being balanced within interface design. Each position within the list was used once in each window. The order of the ten trials was rotated in a Latin square so that after ten participants each trial had been presented in each serial position within the task.

The interface was presented on a $20^{\prime \prime}$ LCD monitor at 1024 $\times 768$-pixel resolution, viewed by participants at a distance of approximately $75 \mathrm{~cm}$. Participants' eye movements were recorded using a SensoMotoric Instruments iView $\mathrm{X}$ eye tracker, recording at $50 \mathrm{~Hz}$, allowing us to count fixations and the total path length of the search on each trail. The presentation computer recorded reaction times for each experimental event.

Results. Reliable eye-tracking data was not obtained for five participants. The remaining 21 participants (18 females) completed a total of 420 trials with three task steps in each trial, of which eye-tracking data was rejected for 89 task steps overall (7\%).

Raw eye-tracking data was analysed using the BeGaze software. The number of fixations, their total path length (expressed as a percentage of the screen width), and the overall duration of each step in each trial were obtained. As the first task step was identical in the two designs, these data were not analysed, giving two task steps in each design.

For each task step, the duration, number of gaze fixations, and total gaze path length as a percentage of screen width were recorded. A MANOVA produced a main effect only for design (Mult: $F_{3,18}=3.92, p=.026, \eta_{p}{ }^{2}=.40$ ), with task step (Mult: $F_{3,18}=2.49, p=.093, \eta_{p}{ }^{2}=.29$ ) and the interaction (Mult: $F_{3,18}=2.72, p=.075, \eta_{p}{ }^{2}=.31$ ) falling short of significance. Univariate $F$ s showed no main effects of design for any of the measures (all $F s<1$ ), but a main effect of task step upon duration, with the third step taking longer $\left(F_{1,20}=5.02, p=.037, \eta_{p}{ }^{2}=.20, \mathrm{MSE}=3.59\right)$. There were interactions of design and task step for fixations $\left(F_{1,20}=8.95\right.$, $\left.p=.007, \eta_{p}{ }^{2}=.31, \mathrm{MSE}=1.04\right)$ and path length $\left(F_{1,20}=\right.$ 4.50, $\left.p=.047, \eta_{p}{ }^{2}=.18, \mathrm{MSE}=.041\right)$, with a marginal effect for duration $\left(F_{1,20}=4.15, p=.055, \eta_{p}{ }^{2}=.17\right.$, MSE $=$ .541). Overall, this pattern of results indicated performance advantages for the translated interface on the second step but for the collocated interface on the third step (Figure 2).

Discussion. Unlike the clear advantage for the collocated map interface [7], there is no real superiority for either interface in this database task. However, there were a number of peculiarities in this task. Participants reported that the alphanumeric code was particularly difficult to recall, especially since the task instructions presented the items in the reverse sequence to that required by the interface. The presence of the Back button, intended to make the designs realistic and allow participants to recover from errors on steps two and three, actually led to an increase in errors compared 


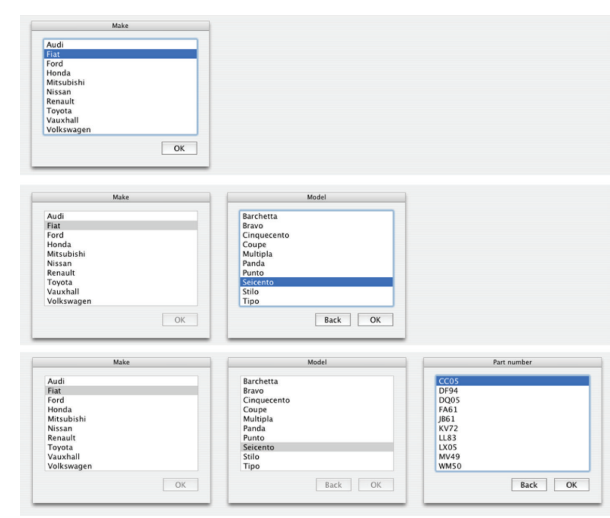

(a)

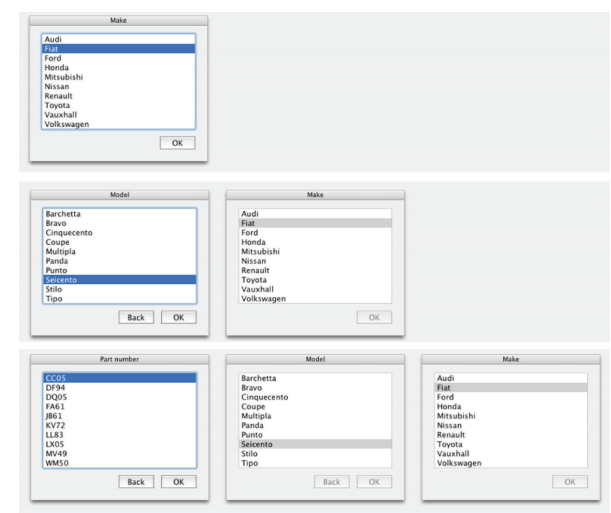

(b)

FIgURE 1: Three successive screens from an interaction with the car-parts database in experiment 1, with the translated interface on (a) and the collocated interface on (b).
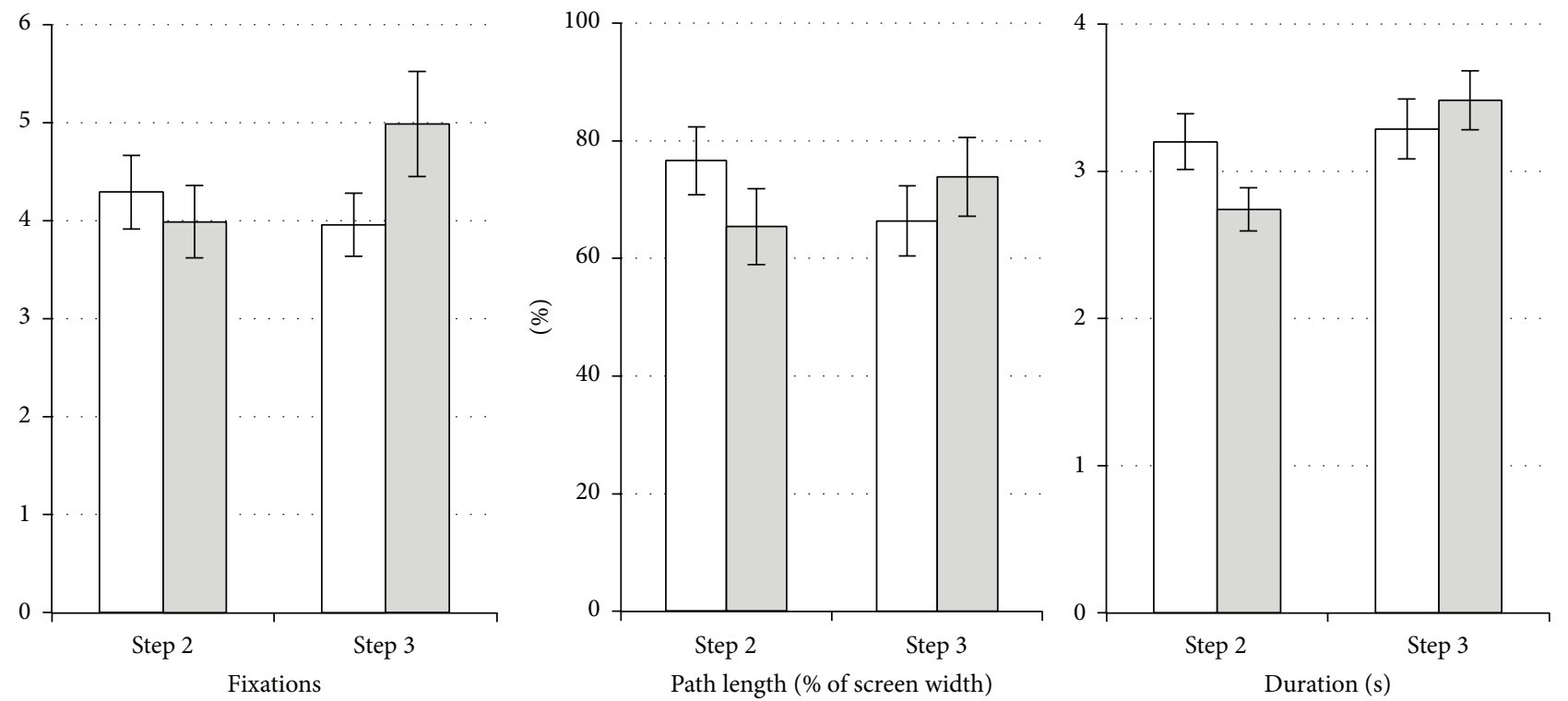

FIGURE 2: In experiment 1, the translated design (grey bars) produced better performance for step 2, but the collocated design (white bars) was better for step 3. Overall, the designs were equivalent. Errors bars show one standard error.

to the first step. Most obviously, though, the reappearance of completed windows to the right of the new window in the collocated interface was unrealistic. While the two designs both displayed the contextual information in the form of previously selected information, a real collocated design would simply replace the old window with the new one rather than moving the old window elsewhere. These issues were addressed in the second experiment.

\section{Experiment 2}

Method. Twenty first year undergraduates from a different large UK university took part in this experiment (age range 20-36 years; median 24 years; 6 males). The design and apparatus of experiment 1 were replicated, with changes to the phrasing of the instructions, the content of the final items, the ordering within the lists, treatment of errors, and the location of the screen in the collocated interface. The meaningless alphanumeric album codes and car parts were replaced with real titles or car parts, and the instructions were reordered so that the items were in the same order as the interface; for example, "please order Fiat - Seicento - fuel filter" or "Please order Jazz - Nina Simone - Ain't got no...."

Within the lists, the items were no longer alphabetically ordered, to avoid participants anticipating a likely location. The Back button was no longer displayed, and if OK was clicked with an incorrect item selected at any point, an error message was displayed and the trial was repeated from the instruction screen. The translated interface window positions were the same as in experiment 1 , with the focal window moving from left to right across the screen leaving the prior windows in their original positions to its left. In the collocated 

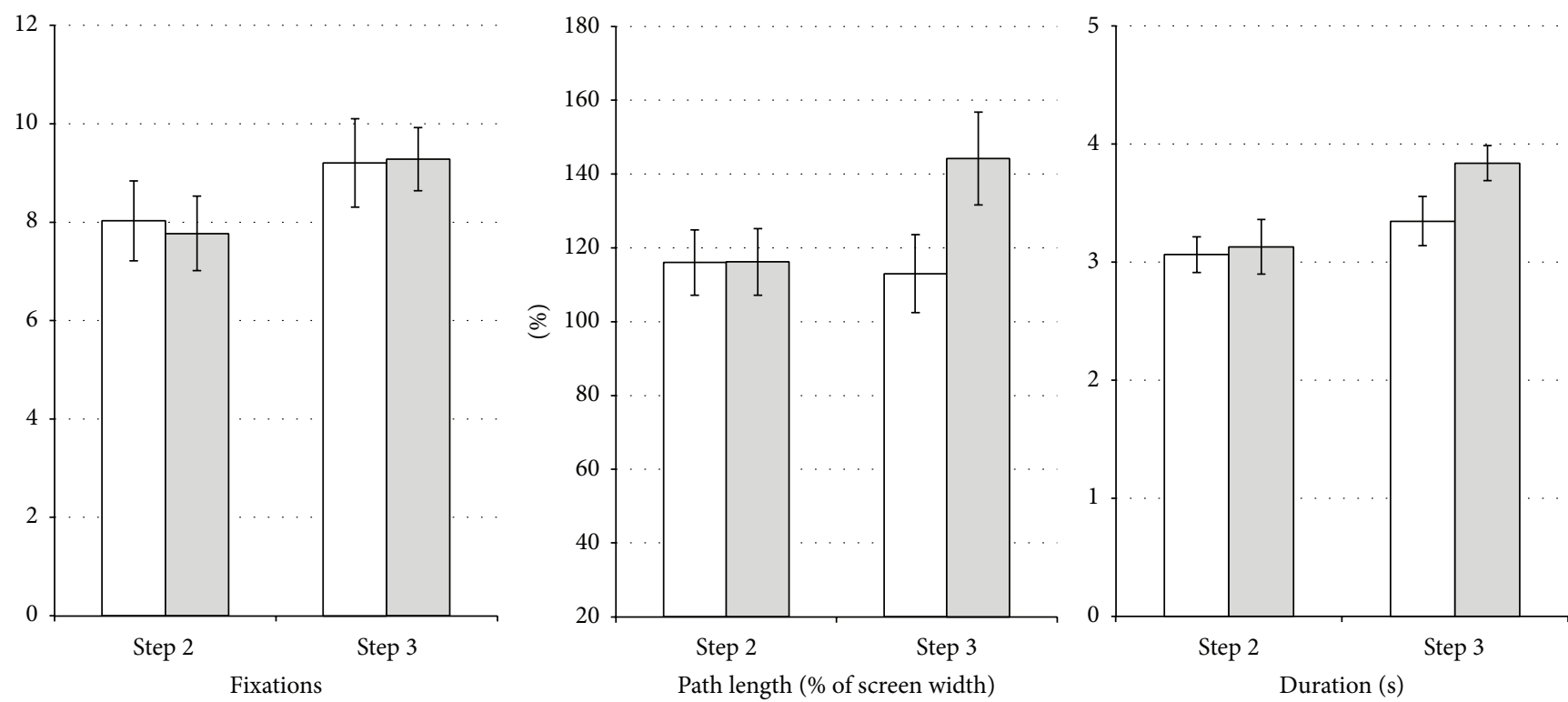

FiguRE 3: In experiment 2, on the second task step the interfaces are indistinguishable, but on the third step the translated interface (grey bars) requires a longer path length and takes longer to complete than the collocated interface (white bars). Error bars indicate standard error.

interface all windows were now located in the centre of the screen, replacing the previous window, so only the current list was visible at any time.

Results. Complete eye-tracking data could not be obtained from four participants. The remaining 16 included 5 males and 11 females.

As in experiment 1 , the duration of the second and third task steps, the number of gaze fixations, and gaze path length were recorded. MANOVA showed main effects of design (Mult: $F_{3,13}=4.92, p=.017, \eta_{p}^{2}=.53$ ), task step (Mult: $F_{3,13}=8.29, p=.002, \eta_{p}^{2}=.66$ ), and an interaction (Mult: $\left.F_{3,13}=10.0, p=.001, \eta_{p}{ }^{2}=.70\right)$. The univariate $F$ s indicated that there were effects of condition upon path length $\left(F_{1,15}=\right.$ $\left.11.1, p=.005, \eta_{p}{ }^{2}=.43, \mathrm{MSE}=.036\right)$ and marginally upon duration $\left(F_{1,15}=4.40, p=.053, \eta_{p}^{2}=.23\right.$, MSE $\left.=.283\right)$ and of task step upon fixations $\left(F_{1,15}=15.7, p=.001, \eta_{p}^{2}=.51\right.$, MSE $=1.84)$ and duration $\left(F_{1,15}=21.5, p<.001, \eta_{p}^{2}=.59\right.$, MSE $=0.18)$, with an interaction for path length $\left(F_{1,15}=17.9\right.$, $\left.p=.001, \eta_{p}^{2}=.54, \mathrm{MSE}=0.022\right)$. The means (Figure 3 ) indicate no difference between the two designs on the second step but an advantage for the collocated interface on the final task step in terms of reduced path length, which results in marginally faster task completion.

Discussion. With the revised interface in which collocated windows replace each other and instructions presenting information in the same order as the task requires it to be used, the two interfaces are indistinguishable for the second task step, but the collocated interface now has an advantage over the translated interface in terms of eye movement path length for the final task step. There is no difference in the number of fixations required and only a marginally significant difference in duration for one of the two steps.
Again, there is no strong advantage in performance for the collocated interface, despite the shorter gaze path lengths required by the design. There is no obvious reason why the translated interface should perform worse in the final step (moving from the artist to the song) than it did on the second step (moving from the genre to the artist), but this is the same pattern as observed in the previous experiment and is worth exploring further.

\section{Conclusion}

This hierarchical database task confirms that the clear benefits of collocation in terms of fewer fixations and faster task completion that had been previously reported in map interfaces [7] are not simply occurring because collocated interfaces require shorter gaze paths. Reductions in gaze path were detected in both experiments, on the final steps, but this was not strongly reflected in the fixation or duration data. While null findings can be difficult to interpret, these two experiments not only show that we did have sufficient power to detect differences between the interfaces but also show that there was no clear overall advantage for one interface design over the other.

Increasing memory load or ambiguity of material, as in experiment 1 , appears to make the contextual information from the first task step more useful and so makes collocation less beneficial, but the information from the second task step is not helping the final task step, where collocation does have some benefit. In hindsight, this may be due to the schematic relationships we drew upon in the experimental materials, which were stronger for the first two task steps but minimal for the third: only Fiat make Seicento cars, for example, but all cars might have fuel filters or "part CC05"; Nina Simone is a Jazz singer, but many artists might record "Ain't got no..." or "track 05AT." 
This is consistent with the underlying cognitive model of film watching $[4,5]$ and its contrast between the "central engine" of cognition required for narrative comprehension, involving high level exchanges between propositional task representations and schematic knowledge, and the lower level exchanges between task representations and visuospatial information required to control visual search [6]. The former would be useful in the second step, but not the final one, in which schematic knowledge is of no help. Just as schematic knowledge can help the viewer of a film predict a novel screen location following a cut, reducing the need for collocation in certain cases, here it removed the benefit of collocation clearly for the second task step, but not for the third.

From a psychological point of view, then, the model of cinematography proposed by May and Barnard [5] can be used to reason about the cognitive consequences of changes to the task. Other applications might include interfaces using verbally ambiguous labels, requiring interactions between task representations and lexical memory stores: here collocation might help reduce referential uncertainty by implying a schematic link between the ambiguous term and the previous task step, although this could also lead to errors if the schematic interpretation is not that intended by the designer. Crucially, our argument is that the link between the principle of collocation and its theoretical derivation allows for extrapolations and extensions like these, which allows guidance to designers to remain current, as novel tasks are digitized and new devices are developed $[8,9]$.

\section{Competing Interests}

The authors declare that they have no competing interests.

\section{Acknowledgments}

This work was supported by the UK Engineering and Physical Sciences Research Council Grant no. EP/C515528/2.

\section{References}

[1] R. N. Kraft, "The role of cutting in the evaluation and retention of film," Journal of Experimental Psychology: Learning, Memory, and Cognition, vol. 12, no. 1, pp. 155-163, 1986.

[2] R. N. Kraft, "Rules and strategies of visual narratives," Perceptual and Motor Skills, vol. 64, no. 1, pp. 3-14, 1987.

[3] E. Young and C. Clanton, "Film craft in user interface design," in Proceedings of the Conference on Human Factors in Computing Systems (INTERCHI '93), Amsterdam, Netherlands, 1993.

[4] J. May, M. P. Dean, and P. J. Barnard, "Using film cutting techniques in interface design," Human-Computer Interaction, vol. 18, no. 4, pp. 325-372, 2003.

[5] J. May and P. J. Barnard, "Cinematography and interface design," in Human-Computer Interaction: INTERACT '95, K. Nordby, P. Helmersen, D. J. Gilmore, and S. A. Arnesen, Eds., pp. 26-31, Chapman \& Hall, New York, NY, USA, 1995.

[6] P. J. Barnard, "Interacting cognitive subsystems: a psycholinguistic approach to short-term memory," in Progress in the Psychology of Language, A. Ellis, Ed., vol. 2, pp. 197-258, Lawrence Erlbaum Associates, Hillsdale, NJ, USA, 1985.
[7] J. May and T. Gamble, "Collocating interface objects: zooming into maps," in Proceedings of the Annual ACM Conference on Human Factors in Computing Systems (CHI '14), pp. 2085-2094, ACM, Toronto, Canada, May 2014.

[8] R. Poppe, R. Rienks, and B. van Dijk, "Evaluating the future of HCI: challenges for the evaluation of emerging applications," in Artifical Intelligence for Human Computing, T. S. Huang, A. Nijholt, M. Pantic, and A. Pentland, Eds., vol. 4451 of Lecture Notes in Computer Science, pp. 234-250, Springer, New York, NY, USA, 2007.

[9] A. Sutcliffe, "On the effective use and reuse of HCI knowledge," ACM Transactions on Computer-Human Interaction, vol. 7, no. 2, pp. 197-221, 2000. 

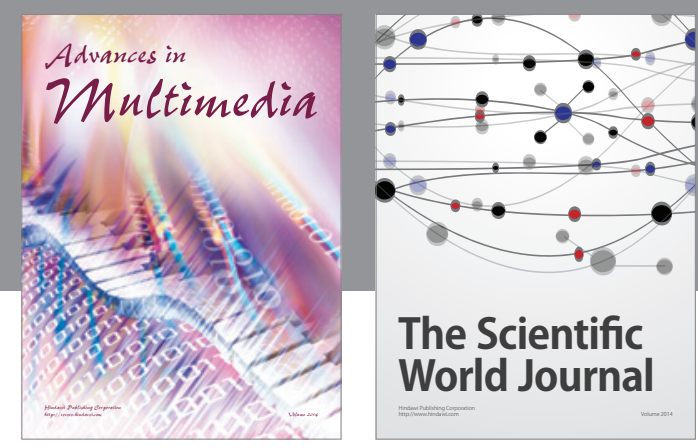

The Scientific World Journal
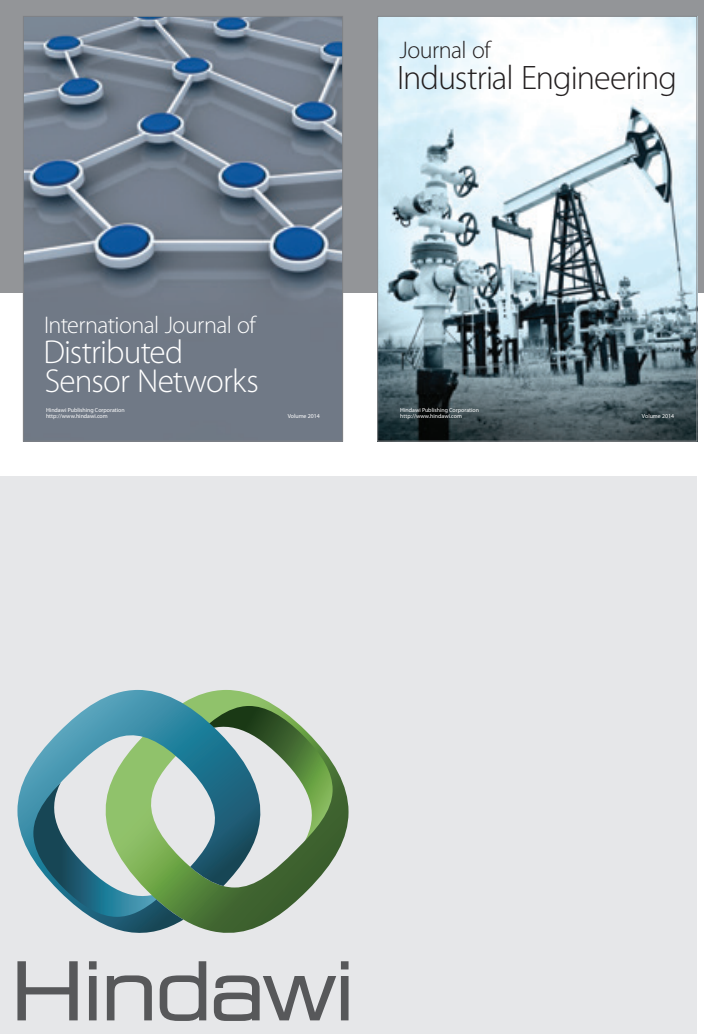

Submit your manuscripts at

http://www.hindawi.com

\section{Computer Networks} and Communications
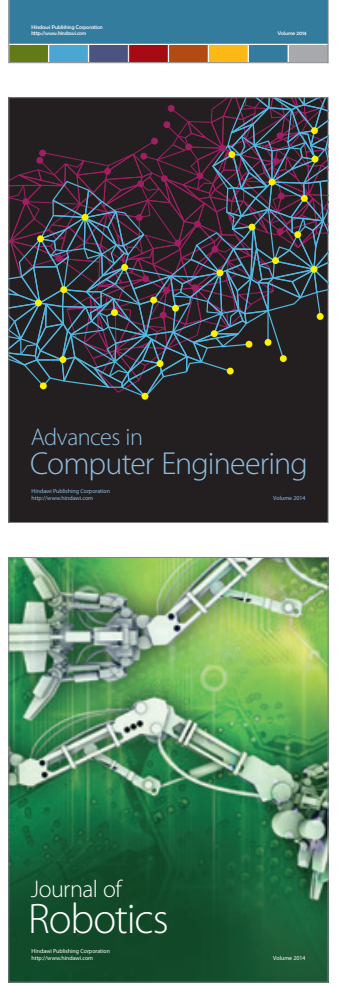
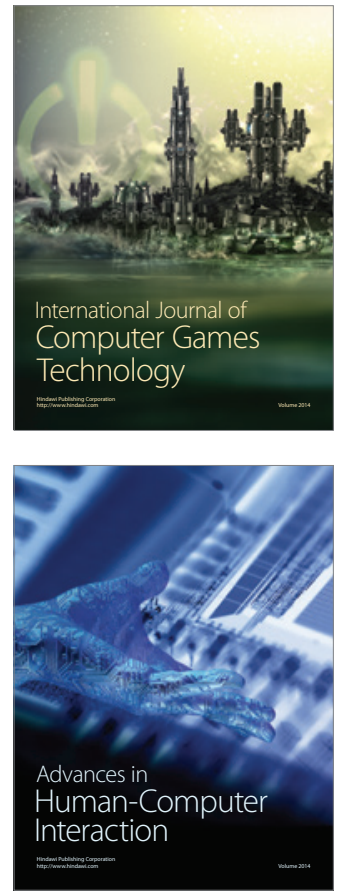
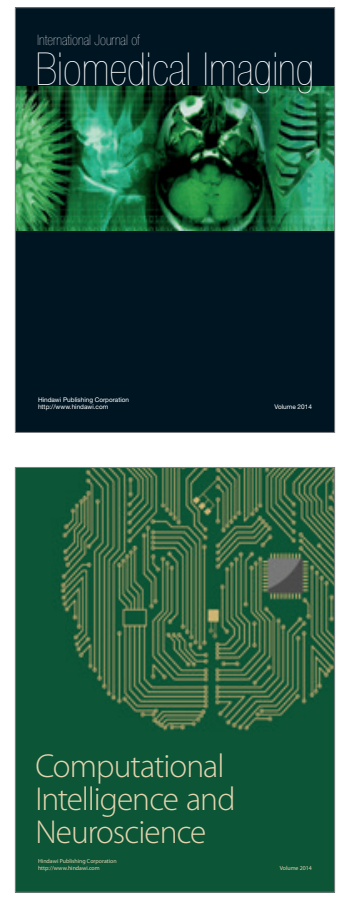
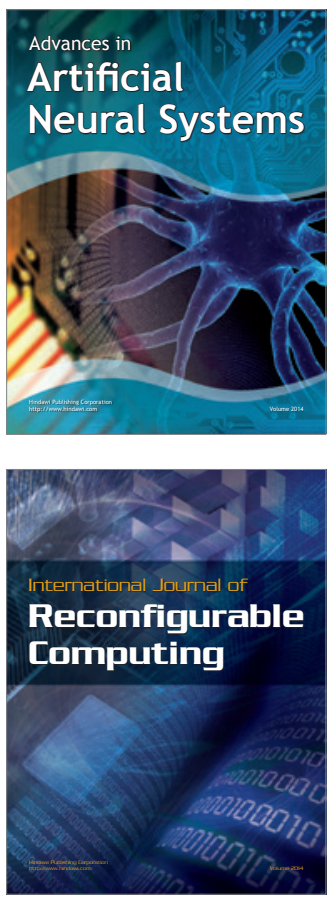
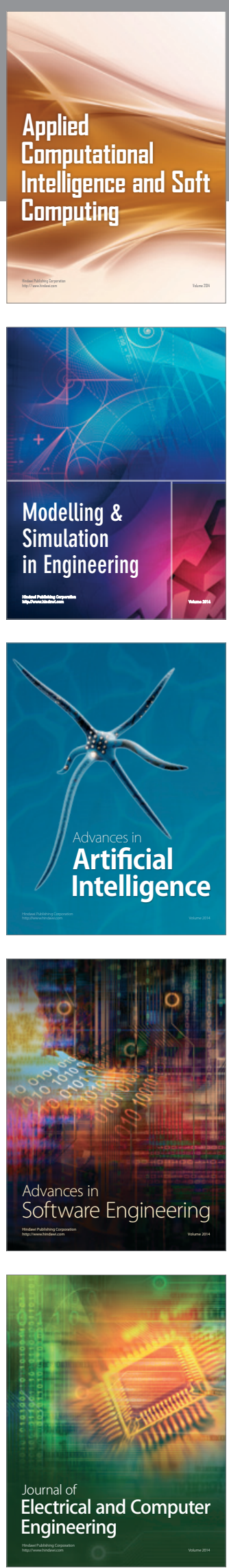